\title{
INTRODUCTION
}

\section{The search for a demography of education: some thoughts}

\section{Bilal Barakat and Hans-Peter Blossfeld*}

At some level, the connection between education and demography is perfectly obvious: indeed, one conceivable definition is that "education is about what one generation believes to be worth passing on to the next". But how can we get from 'Education and Demography' to 'Educational Demography'? Or, indeed, to a 'Demography of Education', and are the latter two the same thing?

While it is generally acknowledged (outside of political grandstanding) that education is inherently a long-term endeavour, the full extent to which this is true is rarely recognised. If we remind ourselves that, in all likelihood, individuals will still be economically active a century (!) from now who will have been taught in school by the very teachers we are training today, it becomes clear that demographic time scales really are relevant to our thinking about education and schooling. This observation is not to say that education policy should attempt to foresee labour market conditions in the distant future - indeed it is debatable whether it should attempt to do so in the present—but it aims to provide a sense of perspective.

Demographic transformations may have educational inputs, such as divergent levels of desired and realised fertility among women of different educational attainment; educational side-effects, for example the consolidation of school networks in areas where the population is shrinking; and educational effects, such as the increasingly diverse classrooms resulting from migration (and in many cases higher migrant fertility). From a different point of view, the roles of inputs and outputs are reversed, if we chose to consider instead the demographic inputs, side-effects and outcomes of the educational process. It is not always obvious that

\footnotetext{
* Bilal Barakat (correspondence author), Vienna Institute of Demography, Austrian Academy of Sciences, Wohllebengasse 12-14, 1040 Vienna, Austria; World Population Program, International Institute for Applied Systems Analysis (IIASA), Laxenburg, Austria. Email: bilal.barakat@oeaw.ac.at

Hans-Peter Blossfeld, Institute of Longitudinal Studies in Education (INBIL), University of Bamberg, Bamberg, Germany.
} 
these phenomena have anything in common that might serve as a 'core' for a Demography of Education.

However, this situation should not serve as an excuse to dismiss the notion altogether. Consider the well-established field of 'Economics of Education'. Here too, the economic perspective can be applied both to educational inputs (the provision of resources) and outcomes (namely private and social returns). Nevertheless, these perspectives can be seen as two sides of the same coin. Indeed both the question "Who benefits?" and "Who pays?" are at the core of economic thinking, and we do not need to fall back on a principle quite as crude as "(S)he who benefits should pay" to recognise that the two questions are closely linked both normatively and in practice. Indeed, current debates in some European countries about university tuition fees financed by taxing graduate salaries vividly demonstrate this link. Where, then, are we to search for the 'essence' of a Demography of Education that conceptually ties together demographic and educational phenomena into a coherent framework?

That the interrelations between demography and education take many forms is reflected in the diversity of the contributions to this issue. Fertility is the subject of four of the ten research articles included here. The next four deal with educational gradients in health and mortality, from a variety of perspectives. The remaining two conversely focus on educational attainment, taking demographic change into account and/or employing demographic techniques. For the convenience of readers, this thematic grouping is reflected in the page order of the contributions.

There are, however, other ways of grouping them. One is to differentiate between those studies that focus on a specific country, and those that are comparative. Among the latter, comparisons between European, sub-Saharan, developing countries worldwide, and a global comparison are represented.

A more profound distinction between the studies may, perhaps, be based on the paradigms, assumptions and ambitions they bring to bear on the study of the connections between education and demography. Obviously no clear-cut categorisation can be applied in this respect. Nevertheless we can observe that some of the contributions are closer in 'spirit' to each other than others, and that these similarities may cut across the geographical and thematic categories mentioned above.

Clearly related in this sense are the contributions by Rios-Neto and Guimarães, and by Spielauer. Both focus on the application of demographic approaches (in the widest sense) to shed light on the educational processes of primary interest. In The demography of education in Brazil: inequality of educational opportunities based on Grade Progression Probability (1986-2008), Rios-Neto and Guimarães disaggregate the expansion in educational attainment in Brazil over the past couple of decades into components representing increased coverage and changes in grade progression, and use these results to test Mare's classical sociological theories regarding inequality in educational 
opportunity. Spielauer, in Persistence and change of the relative difference in educational attainment by ethno-cultural group and gender, uses Statistics Canada's population projection microsimulation model Demosim to examine the dynamics of the relative difference in educational attainment by ethno-cultural group and gender in Canada. It serves as a reminder of the extent to which the education profile of the population depends not only on educational processes themselves but also on composition effects shaped by fertility and migration dynamics.

Examples of such demographic perspectives and techniques are highly welcome, as these are frequently lacking in educational policy debates, even at the highest level. The dangers of neglecting the demographic dimension in formulating educational policy can be clearly seen in examples such as the setting of international development targets for reducing illiteracy without regard for demographic inertia and the way in which this inertia determines which targets can be achieved through improved schooling of the young and generational replacement alone, and which require extensive literacy campaigns directed at adults. Such campaigns would require a massive policy effort.

In fact, education is a process where nothing happens 'by itself' (whether in cognitive learning or in policy). As a result, in education, both at the micro and macro levels there is always a question of rationality in action. The teacher standing in front of a classroom must decide on how to conduct the lesson, even while contradictions in the evidence from cognitive science and psychology on the phenomenon of learning remain unresolved. Similarly, policy choices can rarely, if ever, be delayed until evidence exists that meets scientific standards of being conclusive.

This results in an unavoidable need to develop and to deal with causal heuristics. Not only are the opportunities for randomised experiments extremely rare in the context of population-level educational and demographic phenomena, but in any case, a randomised controlled trial does not necessarily capture what is of greatest policy interest. The latter's pragmatic focus is almost always the marginal effect of exposing those on the threshold of treatment. This requires modelling the (self-)selection process, rather than random experimental assignment. The contributions in this collection struggle with this problem in different ways.

The observation that different fields of study may have starkly different consequences for demographic behaviour, as Bagavos demonstrates in his piece Education and childlessness: The relationship between educational field, educational level, employment and childlessness among Greek women born in 1955-1959, may serve as an example. By itself, we may either interpret this as evidence that it is the content of education that matters. However, we may also conclude the contrary: that having graduated from university has no intrinsic effect, and that it is in fact the disparate socio-economic labour market conditions which graduates of different courses are faced with that account for the 
demographic divergence. Else, it may be neither, but self-selection into academic disciplines.

This does not mean we need fewer such studies, but on the contrary: that we need more, from different countries, and in particular comparative ones. Only by comparing graduates in a given discipline with respect to their selection mechanism, the course contents and work environments they encounter and their fertility relative to graduates from other disciplines and across countries, can we begin to draw sound conclusions.

Focusing on the disaggregation of the demographic outcome, namely parityspecific fertility, rather than the disaggregation of the educational covariate, Van Bavel and Różańska-Putek examine Second birth rates across Europe: interactions between women's level of education and child care enrolment. Here too, the statistical evidence by itself only goes so far but, embedded into a cautious and well-reasoned argument, sheds valuable light on why we should not expect a simple relationship between the availability of child care and fertility by education.

While still shy of proving a casual link, arguments based on covarying educational and demographic behaviour are strengthened if these patterns can be shown to hold over time. Two contributions examine the expansion of education in a European country and relate it to the evolution in fertility, and health and mortality, respectively. A side effect of such an approach is the ability to estimate how much of the change in demography can be attributed to a composition effect, where the level of educational attainment either epitomises or at least indicates membership in the different behavioural groups.

Neels and De Wachter, in Persistent socio-economic differentials in Belgian fertility: can they explain secular trends in tempo and quantum?, argue that educational expansion may account for almost half the change in early fertility between Belgian cohorts born before and after 1950, and also that the education level has to be taken into account in assessing the impact of the economic and policy environment on the tempo and quantum of order-specific fertility.

The findings by Klotz, in Convergence or divergence of educational disparities in mortality and morbidity? The evolution of life expectancy and health expectancy by educational attainment in Austria in 1981-2006, namely that in addition to the composition effect of educational expansion shifting the population into lower-risk groups, the female life expectancy gap decreased at the top, but increased at the bottom end of the educational attainment scale, confirms the suspicion that the education groups are far from homogeneous, and that selection is operating on other characteristics that have health implications.

Taking the above approach and applying it to the problem of projection, $\mathrm{KC}$ and Lentzner look both into the past and the future in their investigation of The effect of education on adult mortality and disability: a global perspective. Their analysis leads to the conclusion that taking the educational composition effect into account, our expectations for disability burden formed purely on chronological 
age have to be adjusted significantly.

It is trivially true that an analysis of (co-)variance (not even in its manifestation as formal statistical tests) does not establish causality. Yet it is certainly not the case that it produces no insights that could be used in a causal argument. The observation that a variable $x$ is an important source of variation in outcome $y$ cannot prove causality because it cannot refute certain kinds of alternative causal explanations (such as a common cause). But neither does it mean it cannot refute any. An important example is that if the residual variance after accounting for educational variation is less than that remaining after accounting for income variation, this pattern is incongruous with the claim that the educational effect is merely an indirect one operating through income alone. Indeed, the formalisation of this type of reasoning is what confirmatory analysis in Structural Equation Models is based on.

Fuchs, Pamuk and Lutz, in their piece Education or wealth: which matters more for reducing child mortality in developing countries? make precisely such a comparison between two competing explanations. They too find that the supposition that education operates on child mortality (in this case) only or mostly through wealth effects does not square with the empirical evidence.

In any case, one researcher's "competing explanation" is another's "mechanism". Even in mathematics, Lakatos (1976) reminds us that the central challenge is not to prove that a result holds, but to clarify under which conditions it does so. The contribution by John Boongaarts, a sweeping review of fertility in sub-Saharan Africa, The causes of educational differences in fertility in subSaharan Africa serves to illustrate this. The title tellingly - and aptly-speaks of "causes of educational differences in fertility", rather than "educational causes..." Flandorfer and Fliegenschnee in their piece Education and health: theoretical considerations based on a qualitative Grounded Theory study investigate how education influences health behaviour.

These two papers employ very different methods, at vastly different geographic scales and levels of measurement. Yet they share a purpose, somewhat distinct from that of the other contributions. Both attempt to elucidate the mechanisms through which education affects demographic behaviour. By uncovering mediating factors through which education operates indirectly, the authors mean to explicate the causal effect of education, not to disprove it.

The objections to crediting formal education with more than a correlative role deserve serious attention. To this end, we have invited three scholars to contribute their thoughts on this controversial question in the form of short argumentative pieces, which add up to a separate debate section in this issue. This debate centres on the following question: If it is an important source of heterogeneity for most processes that population statistics and projections are commonly used for, should educational attainment routinely be used as an additional dimension of disaggregation, alongside age and sex?

The three contributors to the debate come to extremely different conclusions 
on these questions. They are Wolfgang Lutz, whose research groups at the International Institute of Applied Systems Analysis (IIASA) and the Vienna Institute of Demography (VID) have a long record of injecting educational considerations into demographic research and debates, Harvey Graff, who has long warned of the dangers of reifying formal educational attainment, and Alaka Basu, whose extensive demographic fieldwork has put her face to face with both the promises and limitations of education.

To seek out the sources of their disagreement, to what extent it arises from different assumptions, interpretations of the evidence, or of the question, is an enlightening exercise.

On the one hand, the very fact the proposal is controversial may be viewed as an argument against it. Indeed, heated debates around its adoption may distract from any benefits such adoption may otherwise offer. On the other hand, many innovations we take for granted today met initial resistance or even ridicule, and pushing the boundaries of a discipline further necessarily requires moving beyond the existing consensus.

Partly, the disagreement revolves around the question: how much does it matter whether education 'causes' the demographic heterogeneity we often observe, or whether it is merely a signal-as long as it is a strong, consistent signal? If all we wanted to extract from a causal relationship were the assurance that education will remain such a signal in the future, we would be committing a fallacy; for in the social realm, even truly causal relationships are always contingent on contextual conditions that may change and render the effect inoperative at a future point in time.

The demands on a causal connection between education and demographic processes, or vice-versa, are rather different if we wish to use its counterfactual implications for the purpose of evaluating or designing policy.

A case in point are the datasets of reconstructed and projected populations by age, sex and educational attainment prepared at the International Institute of Applied Systems Analysis (IIASA). These data cover 120 countries for the period 1970-2050 and are freely available both from the IIASA website and the World Bank's education database (see the back page in this issue).

This disaggregation has already proven its value. Based on these reconstructions, it is possible to show that the changing educational composition of the population makes a significant difference to projected levels of old-age disability (Sanderson and Scherbov 2010), for example, and calls into question many policy conclusions based on the assumption that in terms of elderly people, there will be "the same, only more of them" in the future. These datasets do not, of course, only represent a disaggregation of population by educational attainment, but conversely also a disaggregation of human capital by age. Using the age information, the positive effect of human capital on economic growth at the national level becomes much clearer (Lutz et al. 2008).

These examples demonstrate that the value of taking the demographic 
composition into account when investigating questions concerning educational attainment, and the educational composition when investigating demographic phenomena, does not hinge on a strictly causal connection. What is key for many purposes is that one carries information regarding the other, information that can be exploited.

Where does this leave us?

In theorising about social phenomena, we can only achieve good simplifications after first having gone through a stage of complexification that may seem daunting and confusing at times. We should therefore not be afraid to 'muddy the waters' as we collect observations on how the education-demography link is contingent: contingent on the content, quality and audience of schooling, on social context, on the economic environment. The list goes on.

We do not share the pessimistic view that no new insights can be gained from building a stockpile of such research, even if by itself some of the pieces may be largely descriptive. Ethnographers begin by collecting 'rich descriptions' of their phenomenon of study; for educational researchers and demographers interested in social reality at the aggregate, rather than individual, level, such a rich description must emerge from observing patterns in statistical data, rather than the direct observation of people.

At the same time, there are some 'low hanging fruit' waiting to be picked. That is to say: research areas that are under-explored but where it can clearly be seen that a demographic perspective can readily illuminate a number of educational policy issues without significant technical problems. One example relevant to developing countries might be the formalisation and quantification of the fact that - where the educational fertility gradient is negative - the education of the average child's parents grows more slowly than the education of the parental cohort as a whole. An example closer to current concerns in many industrialised countries relates to the observed lack of teachers from ethnic minorities that could serve as a role model to under-performing students with an immigrant background. The problem is of course that the recruitment of minority teachers is constrained both by the typically younger age profile of immigrant and ethnic minority populations, and the very legacy of educational deficits. In particular, it may not be possible to make the diversity among teachers representative of that among their students without a massive over-recruitment of ethnic minority students into teacher training, relative to their share among qualified young adults. Suitable projections could provide much-needed insights at which point such constraints may begin to slacken.

We hope that this special issue will encourage demographers and educational researchers alike to take the Demography of Education into account in their thinking so that in the future, issues such as those mentioned above no longer fall between the cracks but on the contrary, are tackled from both sides. 


\section{References}

Lakatos, I. 1976. Proofs and refutations: The logic of mathematical discovery. Cambridge University Press.

Lutz, W., J. C. Cuaresma, and W. Sanderson. 2008. "The Demography of Educational Attainment and Economic Growth". Science 319(5866): 1047.

Sanderson, W. C. and S. Scherbov. 2010. "Remeasuring Aging". Science 329(5997): 1287. 